\title{
Mechanisms of impact and contextual aspects of a dementia special care unit in long- term care: a process evaluation
}

Laura Adlbrecht ${ }^{1,2 *}$, Sabine Bartholomeyczik ${ }^{3}$ and Hanna Mayer ${ }^{4}$

\begin{abstract}
Background: In long-term care, persons with dementia are often cared for in specialised facilities, which are rather heterogeneous in regard to care concepts. Little information is available on how these facilities and care concepts bring about changes in the targeted outcomes. Such knowledge is needed to understand the effects of care concepts and to consciously shape further developments. This study aimed to explore the mechanisms of impact of a specific care concept from a dementia special care unit and the contextual aspects that influence its implementation or outcomes.
\end{abstract}

Methods: Using a qualitative approach to process evaluation of complex interventions, we conducted participating observations and focus groups with nurses and single interviews with ward and nursing home managers. Data were collected from two identical dementia special care units to enhance the contrasts in the analysis of two non-specialised nursing homes. We analysed the data thematically. We conducted 16 observations, three group interviews and eleven individual interviews.

Results: We identified seven themes in three domains related to mechanisms that lead to outcomes regarding residents' and nurses' behaviour and well-being. The themes include the development of nurses'skills and knowledge, the promotion of a positive work climate, adjusted spatial structures, adjusted personnel deployment strategy "dedicated time for activities", promotion of relaxation, of engagement in activities and of engagement in social interaction of residents. The implementation and outcomes of the care concept are influenced by contextual aspects relating to the (target) population and cultural, organisational and financial features.

Conclusions: The study found expected and unexpected mechanisms of impact and contextual aspects. The care concept of the dementia special care unit results in higher levels of relaxation, activities, and social interaction of residents. Its implementation highly depends on the shared understanding of nursing and the skills of the nursing team. Changes in residents' characteristics result in altered effects of the concept.

Trial registration: DRKS00011513.

Keywords: Dementia, Special care unit, Nursing homes, Program evaluation, Theory-driven evaluation

*Correspondence: laura.adlbrecht@ost.ch

${ }^{1}$ Department of Nursing Science, Faculty of Social Sciences, University

of Vienna, Alser Strasse 23/12, 1080 Vienna, Austria

Full list of author information is available at the end of the article

\section{Background}

The majority of persons living in nursing homes have a diagnosis of dementia [1]. Their physical and psychosocial care needs are mostly complex, and in long-term care settings, they often experience a loss in their abilities, freedom, reduction in quality of life and avoidable 
health care service use, such as emergency department visits and hospitalisations that may be preventable by more appropriate nursing and physician care in the nursing homes [1-7]. Whereas the majority of persons with dementia living in nursing homes are cared for in non-specialised facilities, an increasing number live in dementia special care units [1]. Although there is no shared precise definition of dementia special care units (SCUs), an empirical study in Germany found that their main characteristics are a segregative living concept, special qualification in psychogeriatric care and additional funding [8]. Often, these aspects are complemented by a specific built environment and activities adopted to the residents' needs [9]. Substantial research has investigated the impact of SCUs on residents' functional status, neuropsychiatric symptoms, the use of restraints, psychotropic medications, quality of life, engagement in activities and social interaction [10-14]. Although these outcomes have been studied intensively, little is known about how they are achieved, especially as SCUs vary in their characteristics and interventions provided. Variations in characteristics and interventions include architectural features (e.g building specific for residents with dementia, use of exit control systems, number of rooms), financing (e.g. additional funding), professionals (e.g. special qualifications of nurses, continuous presence of registered nurse on night shift), residents (e.g. care degree, dementia diagnosis, number of residents who cannot be mobilized out of the bed, number of residents with psychological diagnosis), dementia policy and interventions (e.g. visitor regulations, recording of preferences, case conferences, evaluation of psychotropic drugs, use of dementia specific assessment instruments, presence of an expert on person-centred care) [15]. Evaluations of SCUs based on a specific theoretical understanding of their mechanisms of impact are lacking. Theories regarding the impact of the physical, social and organisational environment on persons with dementia stress the need for its congruity with a person's abilities and needs [16-19]. However, the specialised care of people with dementia is complex and involves multiple players, structures and processes. Therefore, it is difficult to comprehend how the care concept influences outcomes; though, understanding this relationship is important to further develop specialised care for persons with dementia.

This study focuses on SCUs with a specific care concept and uses a theory-driven evaluation approach to understand the mechanisms triggered by the interventions and how they bring about changes in outcomes. The evaluation is based on a programme theory [20]. In a programme theory a theoretical understanding of the intervention is established by articulating assumptions about the intervention components, processes and desired, as well as undesired, changes [20, 21]. The initial programme theory includes a theory of action that describes the intervention components and a theory of change that describes the mechanisms and outcomes. The care concept is developed for persons with moderate dementia who demonstrate challenging behaviours. Its main features are as follows:

(a) Three small-scale, homelike wards for ten residents, including a kitchen and a living area at the centre, adjoining resident rooms and direct garden access;

(b) Educational interventions (including training in validation on Level 1 and $2^{1}$ ), coaching and supervision sessions for staff members;

(c) A person-centred and emotion-oriented approach in care using Naomi Feil's Validation Therapy [23] that is based on the general principle of validation, the acceptance of the reality of another's experience, unconditional appreciation and acknowledgement of the individual needs. Persons with cognitive impairments are classified in one of four stages on a continuum of dementia (Mal Orientation, Time Confusion, Repetitive Motion and Vegetation). Validation Therapy incorporates a range of specific communication techniques. The approach represents, on the one hand, an attitude in the care of persons with dementia and, on the other hand, it can be used as a structured therapeutic activity on an individual basis or in group sessions [24, 25]; and (d) Personalised non-pharmacological interventions. Challenging behaviour is addressed with relaxing, personalised, and emotion-oriented care to make the residents feel understood and to satisfy their needs. Activating, personalised interventions are used to promote social participation and engagement in purposeful activities. During the daytime, one nurse is specifically assigned to offer activities to residents (such as knitting, cooking, gardening, reading) in groups or at individual level.

According to the theory of change, the main outcomes of the SCU care concept are engagement in purposeful activities, social participation, feeling good at a place and have an impact on residents' affective well-being. The initial programme theory guided the development of the methodology of the process and outcome evaluation [26, 27]. This article addresses the process evaluation of the care concept of the SCU.

\footnotetext{
${ }^{1}$ Validation Training: Validation Therapy is trained in three-day basic courses and certification courses Level 1 to 4 . Level 1 (Validation Worker) lasts 9 months and includes five, 2-day theory sessions, practice phases and supervision sessions. Level 2 courses (Validation Group Leader) last six to 9 months and consist of three, 2-day theory sessions, practice phases and supervision days [22].
} 


\section{Methods}

Aim

This paper reports on the process evaluation of a care concept of the above-described SCU and aims to provide insights into how (mechanisms of impact) and under what circumstances (contextual aspects) the SCU triggers changes in outcomes, either intended or not, regarding residents' and nurses' behaviour and well-being. The results of the process evaluation will help to further understand the logic of the care concept, will check the plausibility of the assumptions described in the initial programme theory, will support the interpretation of the results of the outcome evaluation and will inform the revision of the programme theory. The described care concept was already implemented when the process evaluation started. Therefore, this paper reports only on the mechanisms of impact and contextual factors. The aim of this part of the project is translated into the following research questions:

- What mechanisms of impact (regarding residents' and nurses' behaviour and well-being) are activated by the care concept of the SCU?

- What contextual aspects influence the implementation of the concept and its outcomes?

\section{Design}

The process evaluation of the care concept is based on two key functions of the Medical Research Council's guidance for the evaluation of complex interventions: (a) mechanisms of impact and (b) contextual aspects $[28,29]$. Mechanisms of impact intervene between the delivery of a programme and its outcomes and detail the responses of the target group to the interventions of the programme [28, 30,31]. Contextual aspects of the care concept influence its delivery and/or functioning. Contextual aspects refer to "circumstances in which an intervention is implemented that may interact with the intervention to produce variation in outcomes" and include the organisational setting, cultural practices, characteristics of those delivering or receiving the intervention, political and financial factors [32]. In its guidance, the Medical Research Council suggests that the identification of mechanisms of impact is one of the most important functions of process evaluation and that these assumptions or as they call it "programme theories" are crucial to achieve an advanced understanding of the implementation and functioning of the intervention [29]. This understanding of process evaluation is consistent with the assumptions of Chen's theory-driven evaluation about the function of mechanisms and context [20].
To examine the key assumptions of the initial programme theory and to explore how the programme works, we combined qualitative, deductive and inductive research approaches in our methodology. To incorporate contrasting perspectives that enhance the understanding of the sometimes-elusive mechanisms, we collected data with individual interviews, focus groups and observations in the SCUs that implemented the care concept and in non-specialised nursing homes (NSNHs). To address emerging questions during data collection and analysis, our data collection and analysis periods overlapped, and our data collection instruments were designed as being flexible enough to incorporate the emerging themes [28, 33]. All methods were performed in accordance with relevant guidelines and regulations, to structure our manuscript we used the Consolidated criteria for reporting qualitative research (COREQ) [34].

\section{Setting}

The study was conducted in three nursing homes in rural regions of Austria. One of these nursing homes implemented the care concept under study in two SCUs, whose characteristics are described in the background section. The other two nursing homes are NSNHs where data were collected for contrasting purposes. Both nursing homes consist of three wards, with each being laid out for 40 to 45 residents with and without dementia. No specific care concept for persons with dementia is implemented. However, some nurses selectively and proactively offer isolated dementia care-specific interventions to individual residents. One to three nurses in each ward had been trained in Validation in a three-day basic course (see footnote 1). Furthermore, the nursing homes offer one to two planned group activities per day.

\section{Data collection methods}

We conducted open and participating observations, focus groups and semistructured individual interviews. The observations were guided by the initial programme theory and focused on the mechanisms of impact of the interventions, the achieved outcomes regarding residents' and nurses' behaviour and well-being, and influencing contextual aspects. Residents with dementia and any intervention delivered to them were observed. Detailed descriptions of the interventions, the situations, the reactions of the residents and the context were included in the observation protocol.

In addition, we conducted focus groups with nursing staff members (herein referred to as nurses and representing all qualification levels of the nursing team: registered nurses, nursing assistants and home helpers), as well as semistructured individual interviews with ward managers and nursing home managers. To facilitate 
nurses' reflections on mechanisms, we chose to interview them in groups where they could jointly elaborate on these mechanisms [35]. Conducting focus groups was not possible with the ward and nursing home managers because of their small number. Therefore, we conducted individual interviews with the managers. The development of the interview guides for the group and individual interviews were based on the initial programme theory and the results of the observations. This procedure enabled us to deal with topics arising in the observations or not answered therein (e.g., backgrounds for adaptions of interventions, observed deviations of the proposed mechanisms of impact). The interview guides addressed the following aspects: interventions focusing on nurses, spatial design and layout of the wards, activities and social interactions. For each aspect, the understanding, implementation, mechanisms and effects were discussed.

\section{Sample}

For the observations, we recruited a purposive sample of residents who matched the target group of the care concept. We included residents who had a) a dementia diagnosis, b) regularly showed challenging behaviour and c) received or participated in interventions described in the initial programme theory. Other residents, staff members and visitors were observed when they interacted with the observed residents.

Focus groups were conducted with nurses of the participating nursing homes. Our experience with the development of the initial programme theory showed that nurses may struggle to describe the mechanisms of impact; therefore, we established a purposive sample of nurses who were known for their reflective strength. Further inclusion criteria were that the nurses had worked in the nursing home for at least 6 months and could express themselves well in the German language. Individual interviews were conducted with every ward manager and nursing home manager of the participating nursing homes.

\section{Procedures}

Observation data were collected in two time intervals ( $7 \mathrm{am}$ to $1 \mathrm{pm}$ and $1 \mathrm{pm}$ to $8 \mathrm{pm}$, each with two breaks of 20 min each). Both observation intervals were conducted once in every participating ward, which resulted in eight morning intervals and eight evening intervals. The observing researcher (LA) focused on 5-8 residents during one time interval. Potentially eligible residents were identified and approached by LA and the ward manager. Persons interacting with the focused residents were also observed. Observations took place in the common areas of the wards and in the bedrooms of the focused-on residents. LA tried to maintain a passive role by sitting quietly in the background, only interacting with others when directly approached and keeping interactions short. The observation protocols were handwritten and typed for digital data analysis.

For the individual interviews, the managers were asked directly for their consent to participate. The nurses participating in the focus groups were recruited by the ward managers. During each group interview, four aspects of the interview guide were discussed. In each of the individual interviews with the managers, the aspects corresponding to the expertise of the person were addressed. The focus groups and individual interviews were conducted by LA and TN in meeting rooms within the nursing homes and lasted between 45 and $90 \mathrm{~min}$. The interviews were digitally recorded and then transcribed. Additionally, field notes were taken. Identifying information was replaced by anonymous wild cards (e.g. wild card instead of the real name - Mr. Miller= name of R8). Observations were conducted between March and June 2017, and focus groups and individual interviews were conducted between August and October 2018.

\section{Data analysis}

The data analysis was organised with MAXQDA 2018 and followed the six steps of the thematic analysis by Braun and Clarke [36]. This method enables the identification of semantic and latent topics that are either developed inductively based on data or deductively based on the initial programme theory. First, we familiarised ourselves with the data, reread the transcripts and observation protocols and noted first ideas. In a second step, we systematically coded interesting aspects across the entire data set. Afterwards, (step 3), we looked for common aspects and connections between the codes and gathered them under potential themes. Afterwards, (step 4), we reviewed the consistency of the themes in relation to the coded text and the entire data set. This step had to be done repeatedly as we identified interesting new text passages or codes and themes that needed to be rearranged. Subsequently, (step 5), the themes were refined and named, and relationships between them were described. In the sixth step, we produced the report and a tabular presentation of the themes. Since we included several types of data from different settings in the analysis, we triangulated them in a multi-stage process (see Fig. 1). For each text (observation protocols and interviews), steps 1 and 2 were conducted separately. Steps 3 to 5 were conducted for all the data from one nursing home considering the varying statements in different data sets. Subsequently, we brought the themes from the analyses of the individual nursing homes together by carrying out steps 4 and 5 again for the entire data set. Therefore, we focused on the care concept of the SCU and used the 


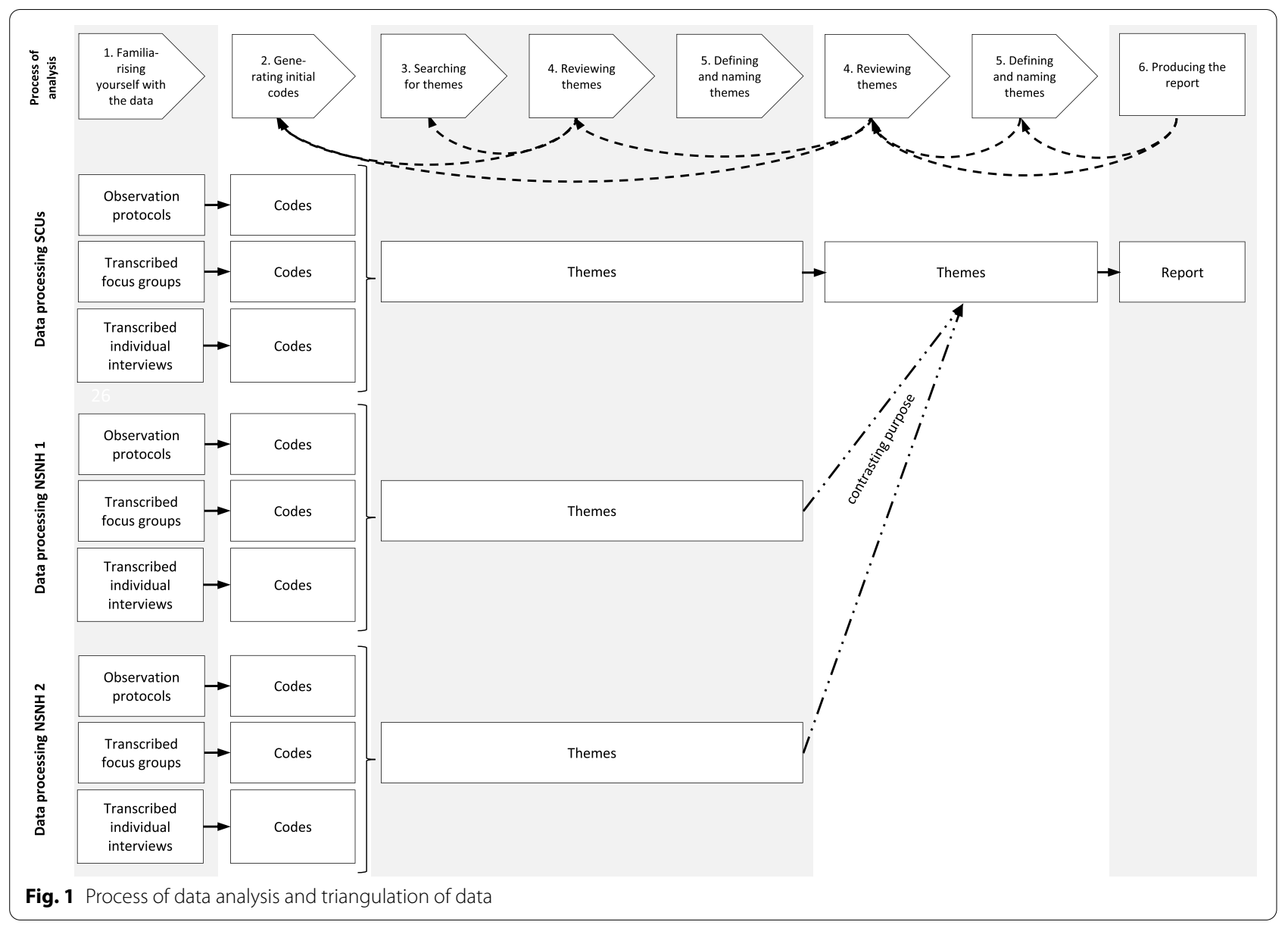

results from the other two nursing homes to contrast them and to gain better insight into the mode of action and mechanisms of impact of the care concept. The coding was performed by LA, and codes and themes were critically discussed in multiple analytic sessions with the co-authors and two further nursing scientists until we reached consensus.

\section{Ethics}

The ethical board of the German Society of Nursing Research approved the study (No. 16-024).

Consent for observations was obtained from all observed residents and all persons possibly interacting with them (e.g. nurses and other residents) before the observation started. Interactions with persons who did not agree to participate in the observations because they did not want to or because there was no opportunity to obtain informed consent beforehand (e.g., visitors who suddenly appeared or nurses from other wards) were not protocolled. To ensure that all participating persons with dementia were able to decide on the highest possible level of information and understanding, they received information about the study adapted to their abilities. Informed consent was obtained from all persons with dementia with the capacity to consent tested with the German version of the University of California, San Diego Brief Assessment of Capacity to Consent [37]. If persons with dementia were not capable to consent, their affirmative agreement was expressed either verbally or non-verbally (assent) [38] and, if designated, their legal representatives' informed consent was obtained. Assent and informed consent were established at the time of enrolment in the study and revisited at each observation session (ongoing consent). Informed consent was obtained from all participants of the focus groups (nurses) and individual interviews (managers).

\section{Trustworthiness}

To enhance trustworthiness, multiple strategies were applied. We triangulated data from different data collection methods and from different sites to test and contrast data and their interpretation. To ensure that participants' statements were accurately captured and to verify our interpretation, we conducted a member check by 
discussing the results with nurses and managers of the SCUs. The translation of the quotes embedded in our article was double-checked by LA and a nursing scientist native in German and English. We know the care concept well, and to reflect our prior knowledge and assumptions critically, we performed parts of step 2 of the thematic analysis [36] in analytical sessions with nursing scientists who were not involved in the study. All researchers have experience with qualitative studies and in research with persons with dementia.

\section{Results}

The results of the process evaluation of the care concept are based on 16 participating observations of 49 residents and 76 persons interacting with them on the wards of the SCUs and the NSNHs, three focus-group interviews with 17 nurses, and eleven individual interviews with ward and nursing home managers (Table 1).

The results regarding the mechanisms of impact were organised into three domains and seven themes. In addition, six contextual aspects influencing implementation and mechanisms of impact of the care concept were identified (see Table 2). The interventions for residents were found to depend on the successful implementation of the interventions for nurses and the spatial and personnel composition of the unit (Fig. 2). This dependency is characterised by the need to develop nurses' skills and knowledge to empower them to carry out interventions for residents. In addition, a positive work climate, spatial structures and an appropriate personnel deployment strategy enable nurses to actually provide interventions to residents adequately. Furthermore, contextual aspects influence the provisioning of the interventions.

\section{Interventions focusing on nurses}

\section{Development of nurses' skills and knowledge}

The development of nurses' skills and knowledge is based on the training of all team members and on collective learning through problem discussion and solving within the team and thereby increases team competence and results in altered and shared care practices (Table 2).

Validation training primarily shapes the fundamental attitude towards care that is expressed by the validation

Table 1 Sample description

\begin{tabular}{|c|c|c|c|c|}
\hline Data source & Total & SCU & NSNH1 & NSNH2 \\
\hline \multicolumn{5}{|l|}{ Observations } \\
\hline Number of observation units, $n$ & 16 & 4 & 6 & 6 \\
\hline Number of observation units in the morning, $n$ & 8 & 2 & 3 & 3 \\
\hline Number of observation units in the afternoon, $n$ & 8 & 2 & 3 & 3 \\
\hline Number of residents focused on in the observations, $n$ & 49 & 16 & 15 & 18 \\
\hline Gender, n women & 38 & 12 & 13 & 13 \\
\hline Age, median (range) & $82(60-98)$ & $82(62-98)$ & $83(60-97)$ & $81(65-95)$ \\
\hline MMSE score, median (range) & $14(0-21)$ & $15(8-18)$ & $13(0-21)$ & $14(3-20)$ \\
\hline $\begin{array}{l}\text { Number of persons observed interacting with the residents focused } \\
\text { on in the observations, } n\end{array}$ & & 17 & 27 & 32 \\
\hline Residents, n & 76 & 9 & 9 & 11 \\
\hline Nurses, n & 29 & 7 & 15 & 20 \\
\hline \multirow[t]{2}{*}{ Visitors (e.g., family members), n } & 42 & 1 & 3 & 1 \\
\hline & 5 & & & \\
\hline \multicolumn{5}{|l|}{ Interviews } \\
\hline Number of individual interviews, $n$ & 11 & 3 & 4 & 4 \\
\hline Number of focus groups, n & 3 & 1 & 1 & 1 \\
\hline \multirow[t]{2}{*}{ Number of persons participating in individual interviews, $n$} & 11 & 3 & 4 & 4 \\
\hline & 3 & 1 & 1 & 1 \\
\hline Number of nursing home managers, $n$ & 8 & 2 & 3 & 3 \\
\hline Number of ward managers, $\mathrm{n}$ & 17 & 6 & 5 & 6 \\
\hline Number of persons participating in focus groups, $n$ & 5 & 2 & 2 & 1 \\
\hline Number of registered nurses, n & 11 & 4 & 3 & 4 \\
\hline Number of nursing assistants, $n$ & 1 & 0 & 0 & 1 \\
\hline Number of home helpers, n & 14 & 0 & 2 & 1 \\
\hline Gender, n women & $45(25-57)$ & $49(38-54)$ & $45(28-57)$ & $39(25-52)$ \\
\hline Age, median (range) & $9(3-31)$ & $11(8-14)$ & $10(4-31)$ & $8(3-22)$ \\
\hline Professional experience in years, median (range) & & & & \\
\hline
\end{tabular}




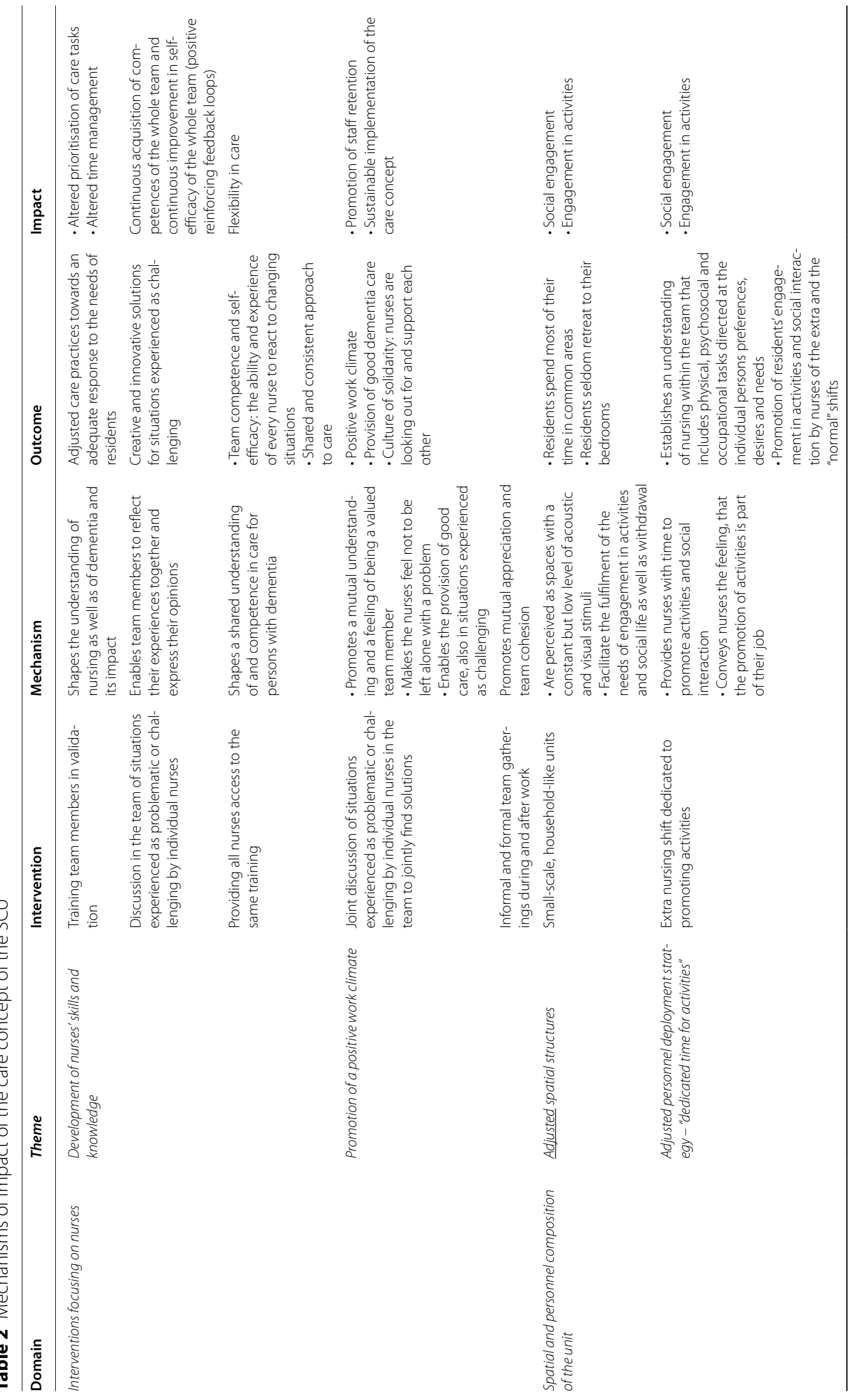




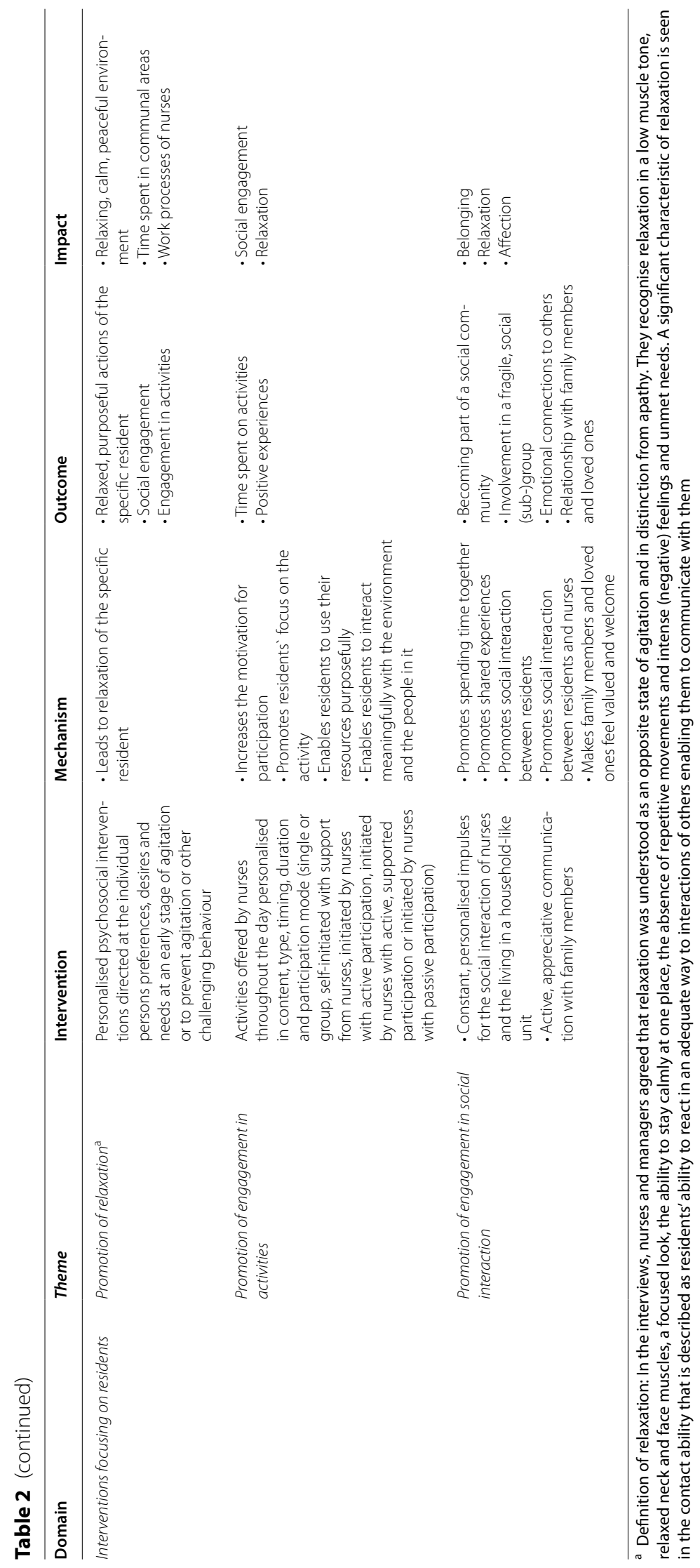


Contextual factors: aspects external to the interventions that affect implementation, mechanisms, or outcomes

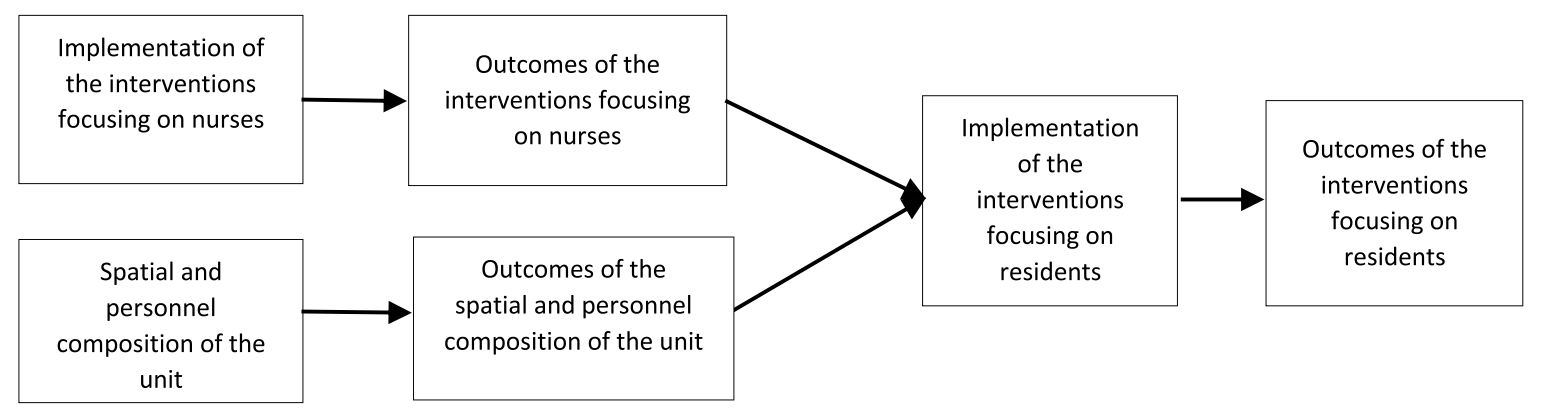

Fig. 2 Relation between interventions and contextual aspects influencing implementation and outcomes of the care concept

of emotions and enhances the amount of empathy shown during care for persons with dementia. Nurses were seldom observed using validation techniques with agitated residents. The focus group participants considered the validating attitude towards care in combination with systematic biographical work as enablers to recognise residents as persons with individual needs, resources, and preferences. Subsequently, nurses described that they are able to orientate their care on the persons' needs, resources and preferences and thereby are able to respond adequately to unmet needs. The observations showed that nurses in the SCUs, compared to those in the NSNHs, use their time resources differently by spending more time on the promotion of activities and social interactions and needing less time to respond to distress and agitation.

Apart from formal training, nurses described that they acquire skills and knowledge by discussing challenging situations with their team. This process was regularly observed with residents showing persistent vocalisations or efforts to run away. Nurses from the SCUs and NSNHs outline their experienced difficulties in informal team gatherings, reflect on their experiences together, discuss opinions and find solutions. Therefore, the team continuously expands and revises its knowledge and skills in dementia care. In an individual interview, a manager described this as follows:

"So that (.) if there are any problems that are very stressful, you also include the team, because the solution usually comes from the team anyway, I have to say. Because if it is a larger group and you discuss, for example, any problem that arises, (.) either we or an individual colleague find a solution." (EI11_58).

As all nurses experience the same development of skills and knowledge, they described that they are more likely to act in synchronisation and thereby offer a consistent approach to care.

"You will make a big leap if you have completed validation training. In addition, above all, as EVERYBODY is trained, we learned to think in the same way." (GI1_28).

As all nurses are trained, everyone has the capacity to react adequately in changing situations, e.g., when a resident acts like she or he never has before. Residents' behaviour was observed to change suddenly and unpredictably; therefore, this team skill is especially useful for preventing stress and agitation. However, the observations in the NSNHs with only a few trained team members revealed that stressful situations may be prolonged if the trained nurses are not on-site.

\section{Promotion of a positive work climate}

A positive work climate is enhanced by collective problem solving, informal and formal team gatherings and mutual appreciation and thereby contributes to the sustainable implementation of the care concept.

The focus group participants reported discussions of difficult situations with the team to jointly find solutions. The discussions made them feel understood, appreciated and not left alone to deal with a problem. They described formal and informal team gatherings as being important for team building and team cohesion. Both problem discussions and team gatherings contribute to a positive work climate and a culture of solidarity, where team members support each other in particularly challenging situations.

\section{"Well, taking care of each other and reminding each} other to take time-outs, for example, helps a lot. If 
there is someone who says, "Let us go smoke one," this only takes five minutes, and then it's alright again. Many of us look out for each other." (EI7_100).

Furthermore, mutual support and jointly developed solutions for particularly challenging situations enable nurses to be more likely to provide good dementia care. The positive climate on the team, the feeling of appreciation and the ability to provide good care reduce nurses' distress. According to the managers of the SCU, this can be seen in high staff retention rates. Staff retention leads to increased efficiency of training measures and higher team competence, which is essential for the sustainable implementation of the care concept.

\section{Spatial and personnel composition of the unit}

The design of the unit and the personnel deployment strategy create spaces, times and an understanding of nursing that promotes activities and social engagement (but also enables privacy and withdrawal) (Table 2).

\section{Adjusted spatial structures}

As pointed out in the interviews with the ward managers, the household-like design of the unit offers spaces that support the fulfilment of residents' needs (e.g., a spatial layout that enables tacit orientation, a patio and a garden with safe walking opportunities, a dining and living area for eating, recreational activities and social life, various sitting options in the indoor patio for tranquillity and resting, a bedroom for privacy). They see a major advantage in the fact that all parts of the unit are in direct sight of the residents and easy to access, thereby enabling residents to autonomously navigate through the unit according to their preferences, desires and needs.

"The background was actually that we can give the residents an intimate space where they can easily find their bearings. That means having FEW people to deal with. In the middle, a living/dining room surrounded by bedrooms. That is, when the resident comes out of his or her bedroom, he is in HIS living room. On a conventional ward, you come into the aisle and must orientate yourself, 'Do I go left, do I go right?' In addition, we just wanted to prevent this additional disorientation." (EI3_4).

The observations yielded a constant but low level of acoustic and visual stimuli in the small-scale units. In the larger units of the NSNH, however, the level of stimuli (e.g., noise level) was noticeably higher, and residents left situations with extremely high levels of stimuli (e.g., mealtimes) quickly. Residents were seen spending most of their time in areas with a comfortable level of stimuli and with opportunities for the fulfilment of their needs (e.g., social interaction in common areas).

\section{Adjusted personnel deployment strategy "dedicated time for activities"}

In addition to the normal nursing shifts, the development team of the care concept established an extra shift, within which nurses' sole assignment is the provision of occupation either in groups or for individual residents. The individual and focus-group interviews with the members of the SCUs show, especially in contrast to the interviews with the members of the NSNHs, that they integrate not only physical and psychosocial nursing care but also the provision of meaningful activities into their understanding of nursing. The change in the understanding is reflected by nurses who enhance engagement in activities in the extra shift and in the normal shifts.

"Are they going to do something by themselves? No. WE have to give them something and then work with and watch them... (name of resident), for example, she does not stimulate herself, so I sit down, ask her something and she blooms while telling me her stories." (GI1_43).

An unintended outcome of the personnel deployment strategy is that nurses recognise residents' great need for engagement in activities and social interaction. The interviewed nurses of the SCUs consider the promotion of engagement in activities and social interaction as their task, and even though they try to use their time optimally, they cannot offer it on an ideal level for all residents. Consequently, they describe having a certain level of distress as they must decide who is going to experience activity promotion and who is not.

\section{Interventions focusing on residents}

The themes a) promotion of relaxation, b) promotion of engagement in activities and c) promotion of social interaction present interlinked bundles of interventions (Table 2). On the one hand, they are interlinked because sometimes the same intervention can provide relaxation, engagement in activity and/or social interaction. An example is a group singing activity accompanied by social interaction that promotes residents' relaxation if it meets their needs. On the other hand, the three complexes are interlinked via their achieved outcomes. Because residents are unique individuals, they respond differently to interventions, allowing the same intervention to achieve different outcomes and different interventions to achieve the same outcomes. Therefore, the three intervention complexes promote the same outcomes, especially relaxation, social engagement and belonging and seem to contribute to the overall well-being. In addition, all three 
intervention complexes are provided in a personalised manner, meaning they are tailored to residents' needs, resources and preferences.

\section{Promotion of relaxation}

For the implementation of the promotion of relaxation (definition in Table 2), nurses primarily use an emotionoriented approach with psychosocial interventions referring to the individual persons' preferences, needs and desires. These interventions are provided as soon as nurses detect a potential for stress, agitation or apathy, but they are also applied for de-escalation purposes. Such interventions allow residents to relax, become contactable, and participate in purposeful activities and social interactions.

Subsequently, the interventions support a calm and relaxed atmosphere on the wards, which was only selectively interrupted by arising restlessness during the observations. Nurses reported that residents usually reflect on the conditions or behaviours, be they calm or agitated, that lead to a reinforcement of the relaxed atmosphere on the ward. A registered nurse described such reinforcement, this time negatively, in the following quote:

\section{"When you come to the ward and you are out of bal- ance, you can be sure that the group is EQUALLY out of balance all day long. They have sensors for that, and if you're having a bad day, you're already screwed. In addition, they notice exactly when you're sad, they look at you and ask, 'What's wrong?' even though you do not do anything differently and think that you're acting quite normal. It works the other way around too; if you come in a good mood, the residents reflect this too." (GI3_161).}

The calm atmosphere is reflected by the low acoustic level, calm movement patterns and purposeful actions of the residents. Supporting the assumption that residents like the atmosphere in common areas, residents were observed spending most of their time there, and withdrawal to one's bedroom was seldom observed in the SCUs. In the NSNHs with a higher noise level, residents with and without dementia tend to seek the quietness of their rooms and use them for relaxation and recreational activities during the observational periods. However, some residents seemed comfortable in high-noise situations that others tended to find stressful - an aspect that reflects the residents' uniqueness.

The observations in the SCUs that contrasted with the observations in the NSNHs showed the early promotion of relaxation results in a lower amount of time needed for calming and reassuring the residents and preventing the development of a negative social dynamic that would in turn affect other residents. Nurses are therefore likely to invest their gained time resources in the promotion of activities and social engagement, which meet residents' needs and further enhance relaxation. In these situations, a positive social dynamic enhancing relaxation and fulfilment of needs could be observed.

\section{Promotion of engagement in activities}

Interventions to promote engagement in activities are provided in organised weekly planned group activities by the elder-care team ${ }^{2}$ and as individual activities by nurses, thereby allowing residents to experience activities throughout the day. During the observations, nurses of the SCUs provided activities to individuals or small groups that were individualised in regard to content, type and duration to meet the physical, cognitive and social abilities, as well as preferences, of the residents. In the interviews, nurses described that this type of activity promotion allows residents to experience activities that they enjoy. This not only creates a positive experience for the residents but also promotes relaxation and focus on the activity, the use of their skills and interaction with their environment.

\section{"They are calmer, they talk much more about it. They also make statements that you would not expect at all, that you do not believe that they still know about. They truly behave differently. They are more communicative, in any case. (.) You have the feeling that they are calmer inside. It is not about their appearance, the fidgeting or something like that, but you have the feeling that they truly give you their full attention and they are calmer for them- selves." (EI4_67).}

Group activities are usually not or less personalised. The observations showed that the reaction of the residents depended on the extent to which the activity offered by the elder-care team corresponded to their needs, abilities, and preferences.

Nurses reported that the effects of activating interventions are usually limited to the time of the activity. An impact on residents' mood, in the sense of verbal expressions, facial expressions or behaviour, was only reported in a few cases. It is questionable, however, whether there truly was no change in mood or whether it was difficult to determine because of the impact of dementia on emotional expression. Often, the mechanism of impact

\footnotetext{
${ }^{2}$ Elder-care (Seniorenbetreuung): The elder-care team consists of individuals with a formal training in the promotion of activities, occupation and companionship for the elderly. In these nursing homes, they provide group activities for the residents on a daily basis scheduled in a weekly plan. They are not part of the nursing staff.
} 
is triggered by an interaction initiated by nurses that is modulated on top of an activity, thereby increasing its scope and impact, as described in the following observation protocol:

N12 turns on the TV and helps residents make themselves comfortable in front of it: "What do you say we watch series $X Y$ now? Yesterday there was (...); let us see how things go on today. What do you think, (name of R8)? Would you like to watch too, (name of R2)? Well then, come and join us. Shall we bet that they kiss today? All those in favour?" "Sure, they will!" (says R8) Two residents lift their arms. "Who's against it?" (B14_p. 6).

\section{Promotion of engagement in social interaction}

Nurses promote engagement in social interaction by starting and guiding conversations or by setting off impulses that are likely to trigger social interactions. Social interaction helps residents to become part of a social community by facilitating a sense of belonging. Becoming part of a social community also happens passively when residents have common experiences that create an emotional connection. This is especially true for residents with advanced dementia, whose opportunities for verbal interaction may be severely limited. The observations made in both settings yielded that the social community is reflected by residents knowing each other and demonstrating behaviours that characterise the social conventions of social groups (e.g., greeting all present when entering the living room, saying goodbye before going to bed, sharing the newspaper and discussing the content, commenting on the weather).

The focus group participants reported that sometimes within the ward, smaller subgroups of two to four people are formed that are primarily based on mutual positive perceptions. The emotional ties of these residents are closer, which is reflected by residents being keen to spend time together (e.g., walking around together, sitting next to each other for certain activities) and by increased interaction between these residents:

"Therefore, for example, we now have a group that has just formed itself. It is an insanely intense group.

They're having a great time. Even the residents, who nobody had accepted before. There were always two ladies who nobody accepted. Now suddenly, the group works with six residents at the table. In addition, each of the ladies demands her own topic of conversation." (GI2_186).

In such subgroups, the residents experience mutual affection, which sometimes manifests itself physically through a hug or stroking of the hand. However, the subgroups are described by nurses as fragile entities that.

“(..) can dissolve unpredictably. In our ward, it went well for a while when two ladies helped to set the table. Then, one of the two ladies got worse and the second one said, 'Well, I am not going to do that alone.' And since then, she just sits there and looks. That's because she did not want to do it alone." (GI2_76).

The promotion of social interaction includes the facilitation of (close) relationships with family members and loved ones. Nurses promote these exchanges with family members; they openly communicate information about the residents and seek the expertise of family members on, for example, the biography of the residents. In this way, family members feel valued, welcome, and integrated and tend to visit residents more often or for longer periods.

"My experience is that if we take the relatives on board right from the start and we are very open in what we say and do [...], I actually have the relatives very much on board, they help out, they deal with the biographical work of their own dad, mum or whatever. In addition, that leads them to truly deal with them. You see that. They simply visit longer, inform themselves about their family member, what happened, how he is?" (IG_EI1_33).

\section{Contextual aspects}

Seven aspects within the context features population, cultural, organisational and financial are found to influence the implementation and outcomes of the care concept. The aspects and corresponding quotes are displayed in Table 3.

With regard to the target population, the managers of the SCUs and NSNHs reported that the population is changing (contextual aspect 1), with more persons with severe dementia, high levels of physical care needs, (severely) limited mobility and psychological symptoms or diagnoses living in nursing homes. Furthermore, they pointed to the increasing number of persons showing disinhibitory behaviours. This population differs from the target population of the care concept, which was originally developed for persons with moderate dementia who demonstrate challenging behaviours, can move around on the ward independently and perform light household tasks. The managers see possible reasons for these changes in the increased competence in home care, which enables persons to stay longer in their own home. For the SCUs, this means fewer residents who correspond to the defined target 


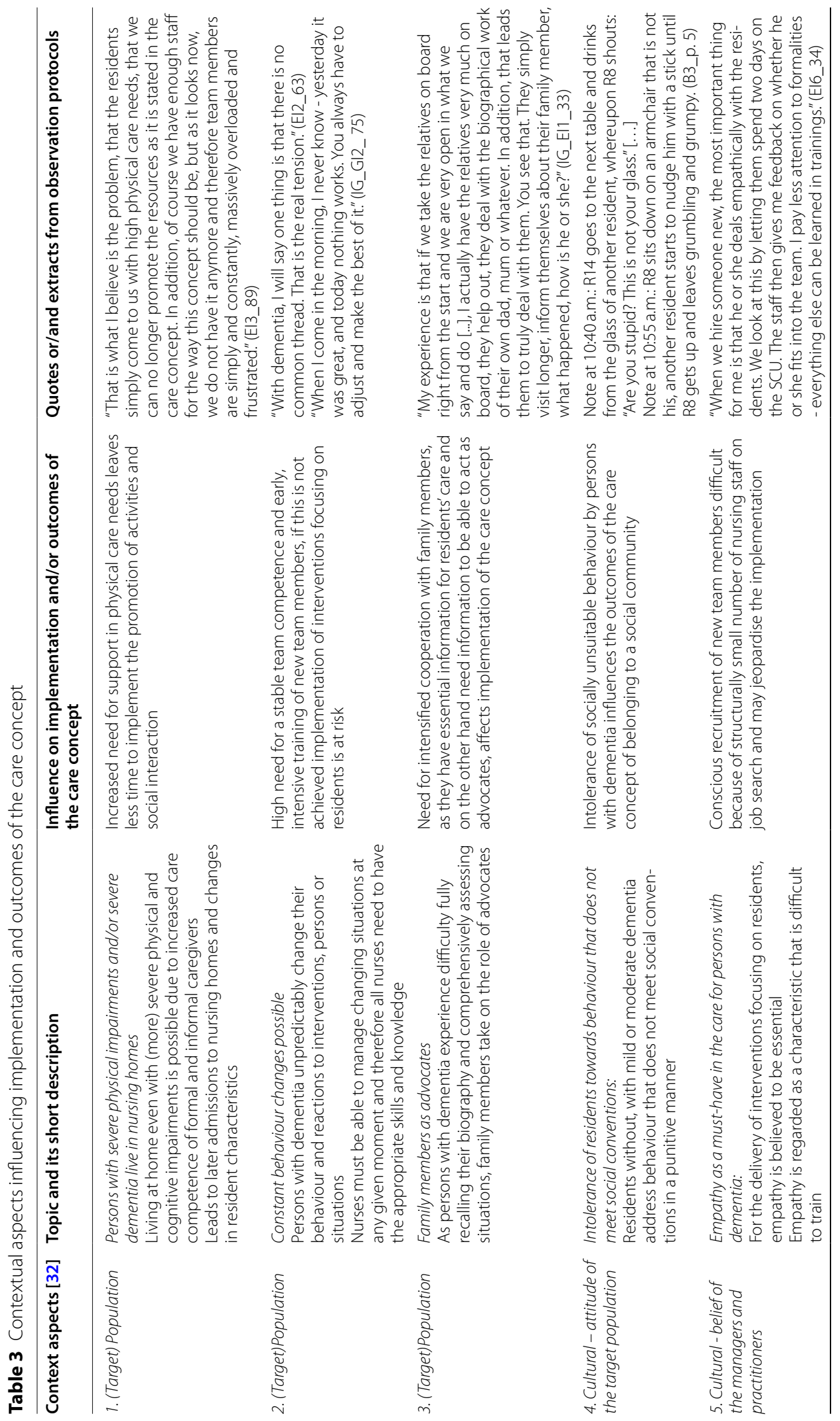




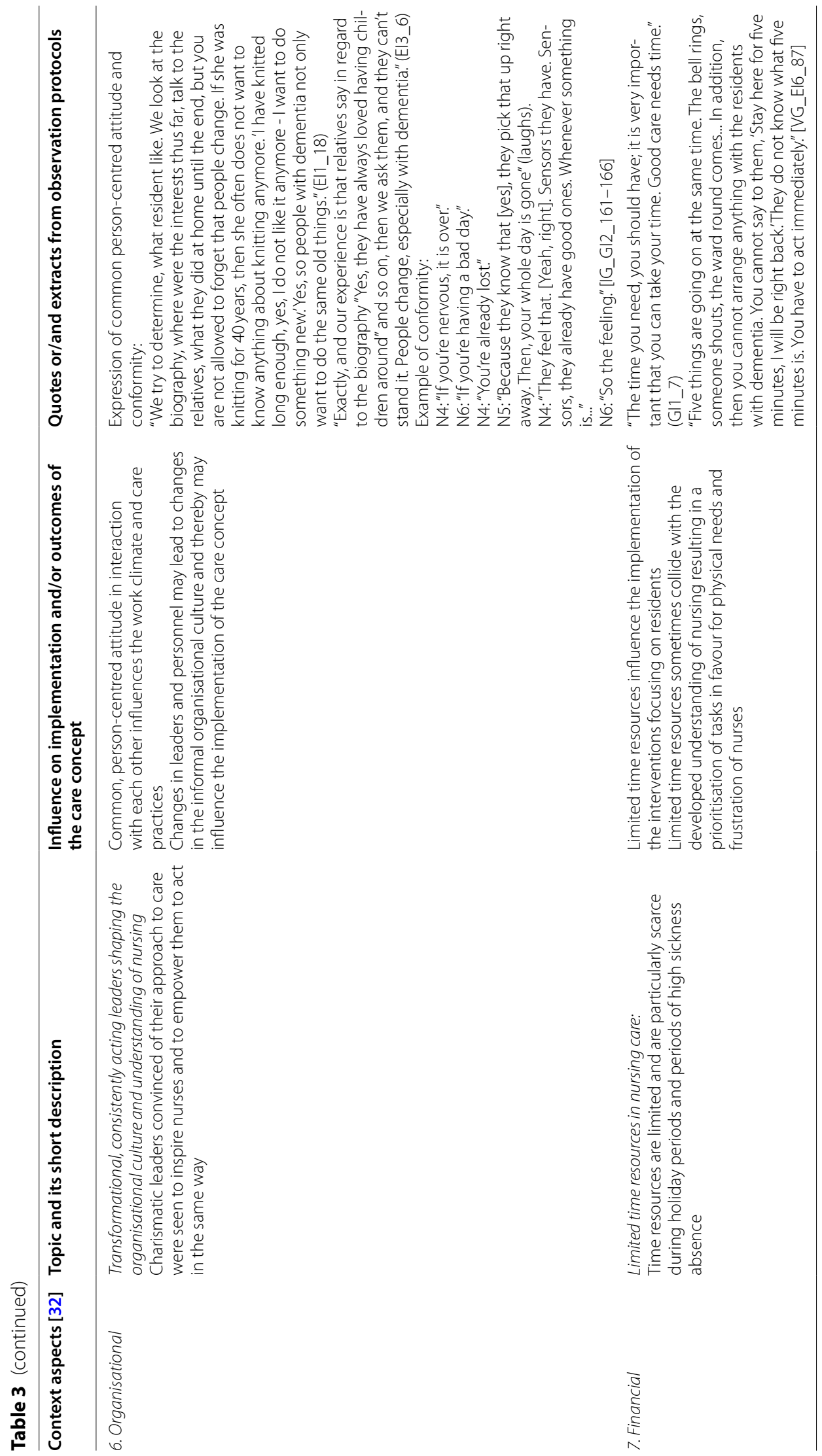


group, more time needed for physical care and less time available for the promotion of engagement in activities and social interaction. In addition, the increased physical and cognitive impairments hinder the promotion of engagement in activities and social interactions in groups requiring individual, time-intensive offers. Another aspect of the target population reported in both settings is that people with dementia often suddenly change their behaviour (contextual aspect 2), which makes the strict adherence to care plans difficult. Nurses must be able to manage changing situations at any given moment. If nurses lack this competence, the unpredictable actions of persons with dementia result in distress for both nurses and residents. An additional aspect of the target population is that due to their impaired memory and decision-making capacity, family members act as their advocates (contextual aspect 3 ), which results in intensified cooperation between family members and nurses.

A cultural aspect of the context observed in both settings is the intolerance of residents towards the behaviour of other residents that is perceived as not being in line with certain social conventions (contextual aspect 4). If a behaviour disturbs residents, they comment on it, sanction it and try to stop it. The observations yielded statements that were often coarse, insulting for the persons referenced and sometimes aggressive. Intolerance towards other residents' behaviours was observed in persons with no, mild, and moderate but not severe dementia. Another cultural aspect is the belief of nurses and managers of the SCUs that care for persons with dementia requires a basic level of empathy (contextual aspect 5), which is difficult to train and essential for the implementation of care based on a person's needs, resources and preferences.

An organisational aspect of context is the transformational leadership style shown by the SCU managers (contextual aspect 6). They are convinced of their approach to dementia care and live their underlying person-centred values in the interactions with residents and nurses, be it in everyday conversations, in organising roasters or in providing support in stressful times. In the observations and interviews, they showed a strong, charismatic appearance and were perceived by the nurses as figureheads. These leaders shape nurses' understanding of respectful interactions with each other and of the care of persons with dementia. In the interviews and in the observations, nurses and managers spoke and worked together consistently, and they were convinced of and enthusiastic about their approach to dementia care. This approach influences the work climate and contributes to a stable team that consistently cares for residents with the same attitude in direct care.
A financial aspect of the context under which care in nursing homes takes place is the limited time resources (contextual aspect 7). Nurses describe having an inner conflict when time resources collide with the developed understanding of nursing that is inherent to the care concept. In such situations, nurses are frustrated and prioritise tasks related to physical needs.

\section{Discussion}

The results of the process evaluation provide insights into how and under what circumstances the care concept of the SCUs triggers changes in outcomes. The findings point to aspects of the initial programme theory that require modification. Table 4 provides an overview of the most substantial indications for the revision. Although the results are not transferable to other settings, as they are linked to the specific care concept and context, the mechanisms of parts of the care concept may also apply in other concepts and in other settings.

Quality of care is influenced by nurses' behaviours that are shaped by the organisational culture and personnel strategy, emphasising a need for sufficient, knowledgeable and skilled personnel [39, 40]. Interventions to promote nurses' expertise and to develop an organisational culture are found to be effective only with a relatively stable team. Staff retention is higher and knowledge drains are lower in supportive workplaces, including adequate staffing levels and working conditions, as well as management support and a person-centred care environment [41-45]. These are aspects to consider, as high staff retention is a key ingredient for the sustainable implementation of the care concept.

In research initiatives, the role of the built environment is often discussed in connection to wayfinding [46] influencing residents' safety and ability to perform activities of daily living, such as toileting or dressing [47]. The findings of the process evaluation indicate that a dementiasensitive spatial design additionally has an impact on residents' social life and their engagement in purposeful and leisure activities, as also shown by Lee et al. [48] and Morgan-Brown et al. [49].

A core element of the care concept is the promotion of activities and social interaction, which address a major need of persons with dementia who want to be involved in activities [50]. The care concept follows the imperative of person-centeredness in dementia care to the extent that the needs, preferences and resources of the residents guide the interventions; however, since it is not systematically implemented at the organisational level, the concept depends on the initiatives of individuals, thereby making it vulnerable to staff or organisational change, which means that it may only contribute to a limited extent to maintaining personhood and continuous 
Table 4 Major indications for the revision of the initial programme theory as implied by the results

\begin{tabular}{|c|c|c|}
\hline Domain & Theme & $\begin{array}{l}\text { Major indications for confirmation, extension } \\
\text { and modification of the initial programme } \\
\text { theory as implied by the results of the process } \\
\text { evaluation }\end{array}$ \\
\hline \multirow[t]{2}{*}{ Interventions focusing on nurses } & Development of nurses'skills and knowledge & $\begin{array}{l}\text { Confirmation: interventions for nurses increase their } \\
\text { competence and communication skills } \\
\text { Modification: interventions for nurses do not reduce } \\
\text { the strain related to dementia care but do increase } \\
\text { self-efficacy } \\
\text { Extension: an increased competence of the entire } \\
\text { team is needed so that nurses can respond flexibly } \\
\text { and adequately to changing resident behaviours }\end{array}$ \\
\hline & Promotion of a positive work climate & $\begin{array}{l}\text { Extension: a positive work climate is regarded as } \\
\text { being essential to maintaining a stable team, which } \\
\text { is needed for sustainable implementation }\end{array}$ \\
\hline \multirow[t]{2}{*}{ Spatial and personnel composition of the unit } & Adjusted spatial structures & $\begin{array}{l}\text { Confirmation: spatial layout reduces stress among } \\
\text { residents } \\
\text { Extension: spatial layout promotes engagement in } \\
\text { activities and social interaction }\end{array}$ \\
\hline & $\begin{array}{l}\text { Adjusted personnel deployment strategy "dedicated } \\
\text { time for activities" }\end{array}$ & $\begin{array}{l}\text { Extension: the important role of the personnel } \\
\text { deployment strategy in shaping the understanding } \\
\text { of nursing and facilitating its implementation only } \\
\text { became clear in the process evaluation; all findings } \\
\text { regarding this theme complement the initial pro- } \\
\text { gramme theory }\end{array}$ \\
\hline Interventions focusing on residents & $\begin{array}{l}\text { Promotion of relaxation, engagement in activities and } \\
\text { social interaction }\end{array}$ & $\begin{array}{l}\text { Confirmation: interventions focusing on residents } \\
\text { promote relaxation, engagement in activities and } \\
\text { social interaction } \\
\text { Modification: interventions focusing on residents, } \\
\text { their mechanisms and outcomes are strongly } \\
\text { intertwined; for example, the same intervention can } \\
\text { promote relaxation and engagement in activity but } \\
\text { can be experienced differently interindividually }\end{array}$ \\
\hline
\end{tabular}

thriving [51-53]. Even though the care concept aims to increase engagement in activities and social interaction, we do not know how much time spent with activities or social interaction each person prefers. Sometimes times of "doing nothing" can be experienced as being relaxing, restorative and especially valuable to residents [54]. Our data are supported by a review of interventions for loneliness showing that the promotion of relaxation, social interaction and activity are intertwined and that each aspect contributes to the others [55].. Furthermore, persons with dementia only marginally seize opportunities to engage socially with others and often rely on nurses to facilitate interactions [56]. Therefore, an understanding of nursing considering the promotion of social interaction as part of nursing contributes to residents' psychosocial well-being by fostering a sense of belonging and significance [57].

The contextual factors are considered complementary to the initial programme theory. Some aspects, e.g., changes in resident characteristics, require changes in implementation that go beyond the adaptive tailoring of complex programmes and can undermine the functionality of the care concept. More severe cognitive and physical impairment, as well as challenging behaviours, are associated with a higher need for direct care time [58]; thus, to be able to maintain the same quality of care, the care concept needs systematic adaption to meet these changing needs [59]. Another contextual aspect that is prone to change is the organisational culture that depends on the leaders and is therefore vulnerable to personnel changes. As a result, person-centred care is not conceived comprehensively in the sense of a process of cultural development that takes into account the care environment, prerequisites and the macro context [52]; thus, its effect cannot reach its full potential [60].

Our results provide useful insights for various stakeholders in nursing homes in the process of planning, managing, controlling and monitoring practice development projects, especially but not exclusively in dementia care. As already mentioned by Lawton [17], the congruence between the elements of a care concept, the context and the needs and abilities of persons with dementia is crucial for achieving desired outcomes. In the care concept studied, the combination of the development of the skills and knowledge of nurses, the physical environment, the nursing time resources, and the leadership style is 
congruent. Our results also show that for the sustainable implementation of the care concept, shared values, and a long-term plan together with a systematic implementation process are beneficial.

The results of the process evaluation that confirm, complement or modify assumptions of the initial programme theory are used together with those of the outcome evaluation to create a revised programme theory [26]. This study shows that complex programmes rely on mechanisms that can be unexpected and that elude initial assessment by researchers. Therefore, it is advisable to include empirical data in the development of programme theories of complex programmes [61, 62]. To further develop the theoretical understanding of dementia care in long-term care, future research should pay more attention to the questions of what works for whom under what circumstances [63] and thereby contribute to an overarching understanding of the active ingredients and their mechanisms of impact of specialised dementia care.

\section{Methodological discussion, strengths and limitations.}

The current study provides an elaborate example of a process evaluation within the approach of theory-driven evaluation in nursing science that focuses on mechanisms of impact and contextual aspects. Even though an increasing number of evaluation studies are incorporating process evaluation, most assess programme fidelity [64-68]; few go beyond this aspect and illuminate the underlying mechanisms of complex programmes. To assess the mechanisms within process evaluation, they use different approaches to theory-driven evaluation, realist evaluation or no theory or methodology at all [69-71]. The care concept of the SCU was developed by practitioners and guided by their experience, and the initial programme theory was based on their assumptions and research evidence. However, the changes, extensions and refinements to the initial programme theory yielded by the process evaluation show how important empirical data from the specific context are for understanding the mechanisms [62]. We gathered data from different contexts, namely, from SCUs and NSNHs, and the constant comparison of the contexts and the interventions, along with a qualitative data-driven research paradigm, enhanced the understanding of the potentially unknown active ingredients of the care concept.

Complex programmes such as care concept of SCUs pose almost infinite uncertainties [72]. Knowing that an all-encompassing assessment is not possible, we followed a pragmatic approach and focused on key uncertainties in our data collection and analysis. Furthermore, the outcomes of an SCU, especially those of higher order, such as belonging, can be attributed to several preconditions, only some of which are influenced by the intervention.
Accordingly, a change in outcome can be caused by the intervention or a contextual aspect. This is because data on outcome indicators should be collected at all stages of the outcome chain of the theory of change [27]. Complex programmes are influenced by infinite contextual aspects. As we could not examine all of the contextual aspects, our study focused on those aspects that showed a substantial influence on the care concept in the inductive research process [32].

Further strengths and limitations of the study are based on the complexity of the care concept, the resulting methodological requirements and the implementation of the concept in only two SCUs. The qualitative inductive approach to process evaluation, which includes different perspectives and data collection methods, is considered a strength that is reflected in the mechanisms that only become apparent in the in-depth exploration of the care concept. Thus, the validity of the results is limited to the context of this care concept. As other SCUs involve other interventions in other contexts, the current results are not transferable but rather provide information about potentially relevant mechanisms, outcomes, and contextual aspects. However, the application of a theorydriven approach to guide the design of an evaluation [26] strengthens the accountability, construct and internal validity of the evaluation [73].

\section{Conclusion}

The current study provides an example of a process evaluation that focuses on mechanisms, outcomes and contextual aspects and thereby assesses how an intervention brings about change. It contributes to understanding what the active ingredients of SCUs are and how they work. The care concept of the SCUs contributes to increased competence and self-efficacy of nurses, and higher levels of relaxation, purposeful activities and social interaction of residents. The implementation of such a concept highly depends on the skills of the nursing team and their shared understanding of nursing practice. As the organisational culture is shaped by individuals and is lacking systematic development, it is comprehended as fragile and vulnerable to unintended changes. Additionally, changes in resident characteristics affect the implementation of the care concept and its outcomes. With further research from different contexts, the findings can be consolidated, and thus, a higher-level understanding of the effects of specialised dementia care can be gained. These findings are important to the further development of dementia care practices on a sound theoretical basis.

\section{Abbreviations}

NSNH: Non-specialised nursing home; SCU: Special care unit. 


\section{Acknowledgements}

We would like to thank all the staff members and managers at the study sites who gave valuable time effort to participate in the study, to support the co-ordination of data-collection and who have shared their insights with us. We thank Tamara Nemeth (TN), a nursing scientist, for her support with data collection. We would also like to thank Mareike Hechinger and Carola Maurer, two nursing scientists, who discussed data and its interpretation critically with us during analytical sessions and thereby enhanced our reflection process.

\section{Authors' contributions}

LA, SB and HM were responsible for the study design. LA collected data. Data analysis and interpretation was performed by LA, SB and HM. LA was responsible for writing a first draft of the paper. All authors substantially revised it critically for important intellectual content and finally approved it.

\section{Funding}

The study was funded by SeneCura, the nursing home operator of the participating nursing homes in the study.

\section{Availability of data and materials}

The datasets used and analysed during the current study are available from the corresponding author on reasonable request.

\section{Declarations}

\section{Ethics approval and consent to participate}

The ethical board of the German Society of Nursing Research approved the study ( $\left.N^{\circ} 16-024\right)$. Consent to participate was obtained of all participants. Details on ethical considerations and the procedure of information and consent is given in the section Ethics.

\section{Consent for publication}

Not applicable.

\section{Competing interests}

The authors declare that even though the study was supported by SeneCura, the funder did not influence the study or its outcomes, and the authors are free to report any unfavourable results. The authors have no conflicts of interest to report.

\section{Author details}

${ }^{1}$ Department of Nursing Science, Faculty of Social Sciences, University of Vienna, Alser Strasse 23/12, 1080 Vienna, Austria. ${ }^{2}$ Department of Health, Center for Dementia Care, Institute of Applied Nursing Sciences, FHS St. Gallen, University of Applied Sciences, Rosenbergstrasse 59, 9001 St. Gallen, Switzerland. ${ }^{3}$ School of Nursing Science, Witten/Herdecke University, Stockumer Strasse 10, 58453 Witten, Germany. ${ }^{4}$ Karl Landsteiner Private University Krems, Dr.-Karl-Dorrek-Strasse 30, 3500 Krems an der Donau, Austria.

Received: 24 May 2021 Accepted: 15 November 2021

Published online: 07 December 2021

\section{References}

1. Alzheimer's association. Alzheimer's Disease Facts and Figures 2020. https://www.alz.org/media/Documents/alzheimers-facts-and-figures.pdf. Accessed 29 Apr 2020.

2. Harris-Kojetin L, Sengupta M, Park-Lee E, Valverde R, Caffrey C, Rome V, Lendon J. Long-Term Care Providers and services users in the United States: data from the National Study of Long-Term Care Providers, 2013-2014. Vital Health Stat 3. 2016:x-xii; 1-105.

3. Hoffmann F, Kaduszkiewicz H, Glaeske $G$, van den Bussche H, Koller D. Prevalence of dementia in nursing home and community-dwelling older adults in Germany. Aging Clin Exp Res. 2014;26:555-9. https://doi.org/10. 1007/s40520-014-0210-6.

4. Førsund LH, Grov EK, Helvik A-S, Juvet LK, Skovdahl K, Eriksen S. The experience of lived space in persons with dementia: a systematic meta-synthesis. BMC Geriatr. 2018;18:33. https://doi.org/10.1186/ s12877-018-0728-0.
5. Nikmat AW, Hawthorne G, Al-Mashoor SH. Quality of life in dementia patients: nursing home versus home care. Int Psychogeriatr. 2011;23:1692-700. https://doi.org/10.1017/\$1041610211001050.

6. Temkin-Greener H, Cen X, Hasselberg MJ, Li Y. Preventable hospitalizations among nursing home residents with dementia and behavioral health disorders. J Am Med Dir Assoc. 2019;20:1280-1286.e1. https://doi. org/10.1016/j.jamda.2019.03.006.

7. Ouslander JG, Lamb G, Perloe M, Givens JH, Kluge L, Rutland T, et al. Potentially avoidable hospitalizations of nursing home residents: frequency, causes, and costs: see editorial comments by Drs. Jean F. Wyman and William R. Hazzard, pp 760-761. J Am Geriatr Soc. 2010;58:627-35. https://doi.org/10.1111/j.1532-5415.2010.02768.x.

8. Bergmann JM, Ströbel AM, Holle B, Palm R. Empirical development of a typology on residential long-term care units in Germany - results of an exploratory multivariate data analysis. BMC Health Serv Res. 2020;20:646. https://doi.org/10.1186/s12913-020-05401-4.

9. Palm R, Bartholomeyczik S. Specialist dementia care units. In: Michel JP, Beattie L, Finbarr M, Watson J, editors. Oxford textbook of geriatric medicine. Oxford: Oxford University Press; 2018. p. 281-5.

10. Lai CK, Yeung JH, MokV, Chi I. Special care units for dementia individuals with behavioural problems. Cochrane Database Syst Rev. 2009:CD006470. https://doi.org/10.1002/14651858.CD006470.pub2.

11. Verbeek H, Zwakhalen SMG, van Rossum E, Ambergen T, Kempen GIJM, Hamers JPH. Effects of small-scale, home-like facilities in dementia care on residents' behavior, and use of physical restraints and psychotropic drugs: a quasi-experimental study. Int Psychogeriatr. 2014;26:657-68. https://doi.org/10.1017/S1041610213002512.

12. Kok JS, Berg IJ, Scherder EJA. Special care units and traditional care in dementia: relationship with behavior, cognition, functional status and quality of life - a review. Dement Geriatr Cogn Dis Extra. 2013;3:360-75. https://doi.org/10.1159/000353441.

13. de Boer B, Hamers JPH, Zwakhalen SMG, Tan FES, Beerens HC, Verbeek $H$. Green care farms as innovative nursing homes, promoting activities and social interaction for people with dementia. J Am Med Dir Assoc. 2017;18:40-6. https://doi.org/10.1016/j.jamda.2016.10.013.

14. Abbott KM, Sefcik JS, van Haitsma K. Measuring social integration among residents in a dementia special care unit versus traditional nursing home: a pilot study. Dementia. 2017;16:388-403. https://doi.org/10.1177/14713 01215594950.

15. Hoffmann AL, Bergmann JM, Mueller-Widmer R, Palm R. Dementia specific care structures in nursing homes-study protocol of a telephonebased survey study in a nationwide random sample. J Adv Nurs. 2021. https://doi.org/10.1111/jan.14873.

16. Weisman GD. Environments for older persons with cognitive impairments. In: Moore GT, Marans RW, editors. Advances in environment, behavior and design. New York: Plenum; 1997. p. 315-46.

17. Lawton MP, Nahemow L. Ecology and the aging process. In: Eisdorfer C, Lawton MP, editors. The psychology of adult development and aging. Washingtion: American Psychological Association; 1973. p. 619-74.

18. Jao Y-L, Liu W, Chaudhury H, Parajuli J, Holmes S, Galik E. Functionfocused person-environment fit for long-term care residents with dementia: impact on apathy. Gerontologist. 2021;61:413-24. https://doi. org/10.1093/geront/gnaa111.

19. Chaudhury $\mathrm{H}, \mathrm{Cooke} \mathrm{H}$. Design matters in dementia care: the role of the physical environment in dementia care settings. In: Downs M, Bowers B, editors. Excellence in dementia care (2nd Edtion). UK: Open Universtiy Press; 2014. p. 144-158.

20. Chen HT. Practical program evaluation: theory-driven evaluation and the integrated evaluation perspective. Los Angeles, London, New Dehli, Singapore, Washington DC: SAGE; 2015.

21. Adlbrecht L, Bartholomeyczik S, Mayer H. Entwicklung einer Programmtheorie als Grundlage für die evaluation einer Demenzstation. [development of a program theory as a basis for the evaluation of a dementia special care unit]. Pflege. 2018;31:125-34. https://doi.org/10.1024/10125302/a000610.

22. Validation Training Institute. Certification Courses Offered by AVOs. 2021. https://vfvalidation.org/education/avo-courses/. Accessed 26 Mar 2021.

23. Feil N. Group therapy in a home for the aged. Gerontologist. 1967;7:192-5.

24. Neal M, Barton WP. Validation therapy for dementia. Cochrane Database Syst Rev. 2003;35:113. https://doi.org/10.1002/14651858.CD001394. 
25. Feil N, De Klerk-Rubin V. Ein Weg zum Verständnis verwirrter alter Menschen. 8. Auflage. München: Ernst Reinhardt Verlag; 2005.

26. van Belle SB, Marchal B, Dubourg D, Kegels G. How to develop a theorydriven evaluation design? Lessons learned from an adolescent sexual and reproductive health programme in West Africa. BMC Public Health. 2010;10:741. https://doi.org/10.1186/1471-2458-10-741.

27. Funnell SC, Rogers PJ. Purposeful program theory: effective use of theories of change and logic models. San Francisco, CA: Jossey-Bass; 2011.

28. Moore GF, Audrey S, Barker M, Bond L, Bonell C, Hardeman W, et al. Process evaluation of complex interventions: Medical Research Council guidance. BMJ. 2015;350:h1258. https://doi.org/10.1136/bmj.h1258.

29. Moore G, Audrey S, Barker M, Bond L, Bonell C, Haredman W, et al. Process evaluation of complex intervnetions. UK Medical Research Council (MRC) guidance 2004. https://mrc.ukri.org/documents/pdf/mrc-phsrn-processevaluation-guidance-final/.

30. Weiss C. Theory-based evaluation: past, present, and future. New directions for evaluations. San Francisco, CA: Jossey-Bass; 1997.

31. Dalkin SM, Greenhalgh J, Jones D, Cunningham B, Lhussier M. What's in a mechanism? Development of a key concept in realist evaluation. Implement Sci. 2015;10:49. https://doi.org/10.1186/s13012-015-0237-x.

32. Craig P, Di Ruggiero E, Frohlich KL, Mykhalovskiy E, White M, Campbell $\mathrm{R}$, et al. Taking account of context in population health intervention research: guidance for producers, users and funders of research. Southampton (UK): NIHR Evaluation, Trials and Studies Coordinating Centre; 2018.

33. Haynes A, Brennan S, Carter S, O'Connor D, Schneider CH, Turner T, et al. Protocol for the process evaluation of a complex intervention designed to increase the use of research in health policy and program organisations (the SPIRIT study). Implement Sci. 2014;9:113. https://doi.org/10. 1186/s13012-014-0113-0.

34. Tong A, Sainsbury P, Craig J. Consolidated criteria for reporting qualitative research (COREQ): a 32-item checklist for interviews and focus groups. Int J Qual Health Care. 2007;19:349-57. https://doi.org/10.1093/intahc/ mzm042.

35. Flick U. An introduction to qualitative research. Thousand Oaks: SAGE; 2014.

36. Braun V, Clarke V. Using thematic analysis in psychology. Qual Res Psychol. 2006;3:77-101. https://doi.org/10.1191/1478088706qp063oa.

37. Jeste DV, Palmer BW, Appelbaum PS, Golshan S, Glorioso D, Dunn LB, et al. A new brief instrument for assessing decisional capacity for clinical research. Arch Gen Psychiatry. 2007;64:966-74. https://doi.org/10.1001/ archpsyc.64.8.966

38. Black BS, Rabins PV, Sugarman J, Karlawish JH. Seeking assent and respecting dissent in dementia research. Am J Geriatr Psychiatry. 2010;18:77-85. https://doi.org/10.1097/JGP.0b013e3181bd1de2.

39. Haunch K, Thompson C, Arthur A, Edwards P, Goodman C, Hanratty B, et al. Understanding the staff behaviours that promote quality for older people living in long term care facilities: a realist review. Int J Nurs Stud. 2021;103905. https://doi.org/10.1016/j.ijnurstu.2021.103905.

40. Blume KS, Dietermann K, Kirchner-Heklau U, Winter V, Fleischer S, Kreidl $L M$, et al. Staffing levels and nursing-sensitive patient outcomes: umbrella review and qualitative study. Health Serv Res. 2021. https://doi.org/10. 1111/1475-6773.13647.

41. Rajamohan S, Porock D, Chang Y-P. Understanding the relationship between staff and job satisfaction, stress, turnover, and staff outcomes in the person-centered care nursing home arena. J Nurs Scholarsh. 2019;51:560-8. https://doi.org/10.1111/jnu.12488.

42. Bratt C, Gautun H. Should I stay or should I go? Nurses' wishes to leave nursing homes and home nursing. J Nurs Manag. 2018;26:1074-82. https://doi.org/10.1111/jonm.12639.

43. Choi J, Kim DE, Yoon JY. Person-centered care environment associated with care staff outcomes in long-term care facilities. J Nurs Res. 2020;29:e133. https://doi.org/10.1097/JNR.0000000000000412.

44. Berridge C, Lima J, Schwartz M, Bishop C, Miller SC. Leadership, Staff empowerment, and the retention of nursing assistants: findings from a survey of U.S. nursing homes. J Am Med Dir Assoc. 2020;21:1254-1259.e2. https://doi.org/10.1016/j.jamda.2020.01.109.

45. Costello H, Walsh S, Cooper C, Livingston G. A systematic review and meta-analysis of the prevalence and associations of stress and burnout among staff in long-term care facilities for people with dementia. Int
Psychogeriatr. 2019;31:1203-16. https://doi.org/10.1017/S104161021 8001606.

46. Kuliga S, Berwig M, Roes M. Wayfinding in people with Alzheimer's disease: perspective taking and architectural cognition-a vision paper on future dementia care research opportunities. Sustainability. 2021;13:1084. https://doi.org/10.3390/su13031084.

47. van Hoof J, Kort HSM, van Waarde H, Blom MM. Environmental interventions and the design of homes for older adults with dementia: an overview. Am J Alzheimers Dis Other Dement. 2010;25:202-32. https:// doi.org/10.1177/1533317509358885.

48. Lee SY, Chaudhury H, Hung L. Effects of physical environment on health and behaviors of residents with dementia in long-term care facilities: a longitudinal study. Res Gerontol Nurs. 2016;9:81-91. https://doi.org/10. 3928/19404921-20150709-01.

49. Morgan-Brown M, Newton R, Ormerod M. Engaging life in two Irish nursing home units for people with dementia: quantitative comparisons before and after implementing household environments. Aging Ment Health. 2013;17:57-65. https://doi.org/10.1080/13607863.2012.717250.

50. Nygaard A, Halvorsrud L, Grov EK, Bergland A. What matters to you when the nursing is your home: a qualitative study on the views of residents with dementia living in nursing homes. BMC Geriatr. 2020;20:227. https:// doi.org/10.1186/s12877-020-01612-w.

51. Kitwood T. Dementia reconsidered: the person comes first. Berkshire: Open Universtiy Press; 1997.

52. McCormack B, McCance T, editors. Person-centred practice in nursing and health care: theory and practice. WileyBlackwell: West Sussex; 2017.

53. Ho P, Cheong RCY, Ong SP, Fusek C, Wee SL, Yap PLK. Person-Centred care transformation in a nursing home for residents with dementia. Dement Geriatr Cogn Dis Extra. 2021;11:1-9. https://doi.org/10.1159/000513069.

54. Harnett T. Framing spaces in places: creating "respite spaces" in dementia care settings. Dementia. 2014;13:396-411. https://doi.org/10.1177/14713 01212474144.

55. Quan NG, Lohman MC, Resciniti NV, Friedman DB. A systematic review of interventions for loneliness among older adults living in long-term care facilities. Aging Ment Health. 2020;24:1945-55. https://doi.org/10.1080/ 13607863.2019.1673311.

56. Adlbrecht L, Bartholomeyczik S, Hildebrandt C, Mayer H. Social interactions of persons with dementia living in special care units in long-term care: a mixed-methods systematic review. Dementia. 2020;1471301220919937. https://doi.org/10.1177/1471301220919937.

57. Kang B, Scales K, McConnell ES, Song Y, Lepore M, Corazzini K. Nursing home residents' perspectives on their social relationships. J Clin Nurs. 2020;29:1162-74. https://doi.org/10.1111/jocn.15174.

58. Vossius C, Selbaek G, Šaltyte Benth J, Wimo A, Bergh S. The use of direct care in nursing home residents: a longitudinal cohort study over 3 years. Int J Geriatr Psychiatry. 2019;34:337-51. https://doi.org/10.1002/gps.5026.

59. Chen EK, Reid MC, Parker SJ, Pillemer K. Tailoring evidence-based interventions for new populations: a method for program adaptation through community engagement. Eval Health Prof. 2013;36:73-92. https://doi. org/10.1177/0163278712442536.

60. Hamiduzzaman M, Kuot A, Greenhill J, Strivens E, Isaac V. Towards personalized care: factors associated with the quality of life of residents with dementia in Australian rural aged care homes. PLoS One. 2020;15:e0233450. https://doi.org/10.1371/journal.pone.0233450.

61. Handley M, Bunn F, Goodman C. Supporting general hospital staff to provide dementia sensitive care: a realist evaluation. Int J Nurs Stud. 2019;96:61-71. https://doi.org/10.1016/j.jpurstu.2018.10.004.

62. Handley M, Bunn F, Lynch J, Goodman C. Using non-participant observation to uncover mechanisms: insights from a realist evaluation. Evaluation. 2019;135638901986903. https://doi.org/10.1177/1356389019 869036.

63. Pawson R, Tilley N. Realistic evaluation. London: SAGE; 2010.

64. Roberts S, Grealish L, Williams LT, Hopper Z, Jenkins J, Spencer A, et al. Development and process evaluation of a complex intervention for improving nutrition among hospitalised patients: a mixed methods study. Healthcare (Basel). 2019. https://doi.org/10.3390/healthcare70200 79.

65. Anrys P, Strauven G, Roussel S, Vande Ginste M, de Lepeleire J, Foulon V, et al. Process evaluation of a complex intervention to optimize quality of prescribing in nursing homes (COME-ON study). Implement Sci. 2019;14:104. https://doi.org/10.1186/s13012-019-0945-8. 
66. Lichtwarck B, Myhre J, Selbaek G, Kirkevold Ø, Rokstad AMM, Benth JŠ, et al. TIME to reduce agitation in persons with dementia in nursing homes. A process evaluation of a complex intervention. BMC Health Serv Res. 2019;19:349. https://doi.org/10.1186/s12913-019-4168-0.

67. Luker JA, Craig LE, Bennett L, Ellery F, Langhorne P, Wu O, et al. Implementing a complex rehabilitation intervention in a stroke trial: a qualitative process evaluation of AVERT. BMC Med Res Methodol. 2016;16:52. https://doi.org/10.1186/s12874-016-0156-9.

68. Bridges J, May C, Fuller A, Griffiths P, Wigley W, Gould L, et al. Optimising impact and sustainability: a qualitative process evaluation of a complex intervention targeted at compassionate care. BMJ Qual Saf. 2017;26:9707. https://doi.org/10.1136/bmjqs-2017-006702.

69. Silies K, Schnakenberg R, Berg A, Kirchner Ä, Langner H, Köberlein-Neu J, et al. Process evaluation of a complex intervention to promote advance care planning in community-dwelling older persons (the STADPLAN study)-study protocol. Trials. 2020;21:653. https://doi.org/10.1186/ s13063-020-04529-2.

70. Rycroft-Malone J, Seers K, Eldh AC, Cox K, Crichton N, Harvey G, et al. A realist process evaluation within the facilitating implementation of research evidence (FIRE) cluster randomised controlled international trial: an exemplar. Implement Sci. 2018;13:138. https://doi.org/10.1186/ s13012-018-0811-0.

71. Al-HadiHasan A, Callaghan P, Lymn JS. Qualitative process evaluation of a psycho-educational intervention targeted at people diagnosed with schizophrenia and their primary caregivers in Jordan. BMC Psychiatry. 2017;17:68. https://doi.org/10.1186/s12888-017-1225-2.

72. Moore GF, Evans RE, Hawkins J, Littlecott H, Melendez-Torres GJ, Bonell $C$, et al. From complex social interventions to interventions in complex social systems: future directions and unresolved questions for intervention development and evaluation. Evaluation. 2019;25:23-45. https://doi. org/10.1177/1356389018803219.

73. Coryn CLS, Noakes LA, Westine CD, Schröter DC. A systematic review of theory-driven evaluation practice from 1990 to 2009. Am J Eval. 2011;32:199-226. https://doi.org/10.1177/1098214010389321.

\section{Publisher's Note}

Springer Nature remains neutral with regard to jurisdictional claims in published maps and institutional affiliations.

- fast, convenient online submission

- thorough peer review by experienced researchers in your field

- rapid publication on acceptance

- support for research data, including large and complex data types

- gold Open Access which fosters wider collaboration and increased citations

- maximum visibility for your research: over $100 \mathrm{M}$ website views per year

At BMC, research is always in progress.

Learn more biomedcentral.com/submissions 